\title{
W-LEACH Decentralized: Weighted LEACH with a Decentralized way
}

\author{
${ }^{1}$ Abdelhalim HNINI, ${ }^{2}$ Abdellah EZZATI, ${ }^{3}$ Mohammed FIHRI, \\ ${ }^{4}$ Abdelmajid HAJAMI \\ ${ }^{1,2,3,4}$ LAVETE laboratory Mathematics and Computer Science Dept Sciences and Tech FacSettat, 26000, \\ Morocco
}

\begin{abstract}
A mobile WSN is considered as a collection of wireless mobile nodes and a base station forming an ad-hoc network. This type of network is used in various areas; such as underwater and underground. Each node has a limited energy and a random mobilization. Since the energy consumption is a very important factor, the Hierarchical protocols can solve this problem for instance the LEACH protocol. W-LEACH is a LEACH extension which extends the network lifetime but this protocol uses sensor location to organize the network so it is not effective in an area where the location of nodes is impossible with mobility. In this paper, we improve an old algorithm (W-LEACH) to a new protocol called W-LEACH Decentralized to increase network lifetime without the use of maps. Besides, we are going to compare this protocol with LEACH in term of network lifetime and energy dissipation using Network Simulator.
\end{abstract}

Keywords: W-LEACH, W-LEACH Decentralized, WSN.

\section{INTRODUCTION:}

Networks of wireless sensors are used in various fields, including monitoring, environment, industrial monitoring, medical applications and disaster response. Structure deployment of sensors and the way of their mobility require a different approach to networking and communication systems compared to the traditional networks. Wireless Sensor Network is composed of a base station and lots of sensor nodes that are deployed in an area. Each node has an objective to sense data and sends it to the BS. These nodes can be deployed in dangerous areas. As a result, the source energy replacement is not feasible because these nodes are inaccessible to the user since the energy efficiency is a key to their performance. Therefore the network should be enhanced to improve the entire network lifetime. The main objective is to balance the energy consumption between all sensor nodes to extend the lifetime of the network so that there would be no overflow sensor nodes used to run out of energy before the others. Generally, the energy consumption by a sensor node integrated sensing, communication and data processing. Among the three operations, a sensor node expends the maximum energy in the data communication. A major concern is the design and development of communication protocols wish to recover energy in order to extended network lifetime.

A practical design scheme of nodes in WSN, It is clustering. This can stabilize the network topology periodically and efficiency at the energetic consumption. LEACH (Low Energy Adaptive Clustering Hierarchy Aggregation) is an increase network lifetime, but this protocol is not effective with mobility. An extension of LEACH is developed, called LEACH-Mobile to support the frequent mobility, in terms of packet loss. CBR Mobile in WSN is also another study to master the management of packet loss but these protocols are consume a lot of energy.

W-Leach is a centralized algorithm. As LEACH, an algorithm operates in cycles so that each tower should have two phases, namely a setting phase and a steady state phase. In the configuration phase, W-LEACH selects a number of sensors to be $\mathrm{CHs}$. Each $\mathrm{CH}$ is selected based on a weight value which corresponds to each node. Unlike LEACH, whether a sensor is $\mathrm{CH}$ or not in the preceding rounds, is independent in W-LEACH. After all CHs are selected clusters are formed such that each sensor is assigned to its closest $\mathrm{CH}$. Leach requires each $\mathrm{CH}$ collects data from all sensors in its group at its steady state phase, against $\mathrm{W}$-Leach chooses only $\mathrm{x} \%$ of sensors in each group to send data to their $\mathrm{CH}$. Candidates for sending data to $\mathrm{CHs}$ are selected according to their weight.

Although the W-LEACH protocol has advantages in terms of an energy recovery but this protocol is based on knowledge of the location of nodes to select the nodes between transmission nodes and nodes remain in sleep. In addition it does not consider the mobility of sensor nodes after the "installation phase" of groups in a round while the W-LEACH protocol accompanies a lot of data loss in mobility environment focused on "hot spots". Because its application is limited to nodes fixed sensors.

In this work the W-LEACH Decentralized outperforms W-LEACH in terms of environment, field of use in underground and Mobility to extended Network lifetime. W-LEACH Decentralized is based on a number 
of neighbors to reorganize node density after that it selects active nodes and those which remain asleep in a decentralized manner.

Our work is organised in the following way: section 1 introduces the WSNs. Section 2 presents related works on the hierarchical routing protocol and the existing LEACH descendent. Section 3 includes the presentation of our contribution. Section 4 shows the simulation steps and demonstrates the simulation results. The last section concludes our work.

\section{LEACH ALGORITHM}

A LEACH protocol is discovered by Heinzelman, to extend the network life time of WSN and to decrease the consumed energy, [1][2]. LEACH (Low Energy Adaptive Clustering Hierarchy Aggregation) algorithm is a data aggregation algorithm that balances the energy among nodes. LEACH is made up of node clusters; each cluster has a particular node as cluster head. The Cluster head collects data from nodes that belong to the respective cluster and sends it to the base station. The $\mathrm{CH}$ role changes periodically. The communication time is divided to rounds. Each round time contains two phases namely, a setup phase and a steady state phase.

In the setup phase, the cluster heads are chosen and clusters are formed. Each sensor is elected to be a cluster head with a probability and only one $\mathrm{CH}$ is elected in one cluster based on a threshold:

$$
\mathrm{T}(\mathrm{n})=\mathrm{p} /\left\{1-\mathrm{p}^{*}(\mathrm{r} \bmod 1 / \mathrm{p})\right\} \quad \text { if } \mathrm{n} € \mathrm{G}(1)
$$

Where $p$ is the desired number of $\mathrm{CHs}, \mathrm{r}$ is the current round, and $\mathrm{G}$ is the set of nodes that have not been $\mathrm{CHs}$ in the last 1/P rounds. That can extend the network life time because the current $\mathrm{CH}$ is not chosen in the next round until all the other sensors in the network become CHs.

In the steady state phase, in cluster LEACH is based on Time Division Multiple Accesses (TDMA) to communicate and transmit data [1]. The same is presented in figure 1, where the round is divided to frame times. Every frame is subdivided to Time Slot which corresponds to one node member to send its data to $\mathrm{CH}$.

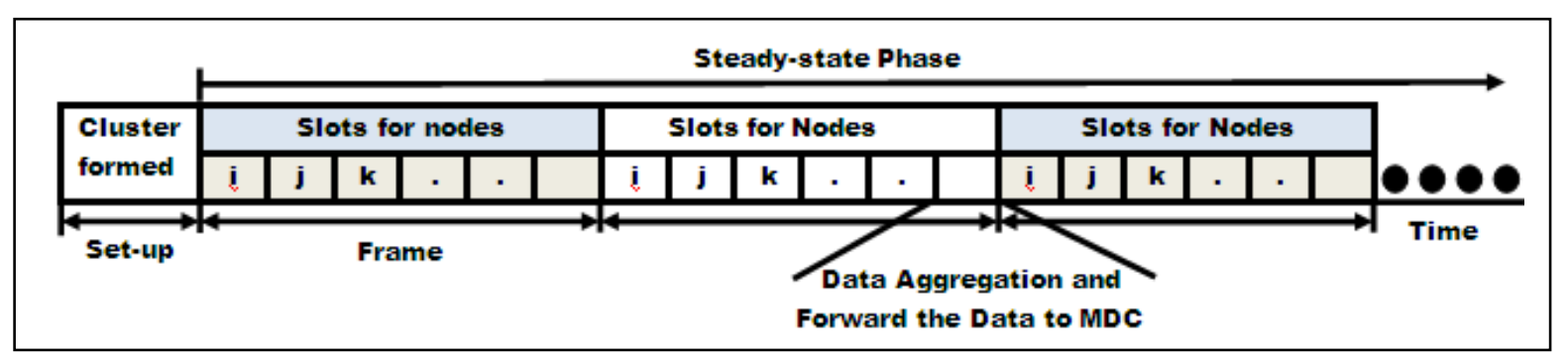

Figure 1: chronologique communication in LEACH

LEACH-C is a modified version of LEACH [2]. The difference between them is at the selection of CHs. LEACH-C is centralized but LEACH is decentralized. In every round, the base station determines the nodes which are CHs. It focuses on node locations.

The Figure 2 presented LEACH and LEACH-C aggregation algorithms.

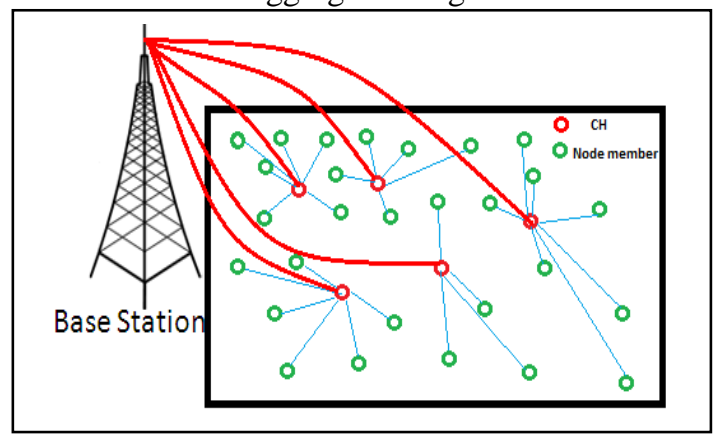

Figure 2: LEACH and LEACH-C aggregation algorithm

\section{A. LEACH mobile and CBR-LEACH algorithms}


A lot of studies are published to improve LEACH protocol and LEACH-C in term of energy dissipation and $\mathrm{CHs}$ dies as in [3][4].

Other protocols have improved LEACH in terms of mobility and packets loss. These protocols types are similar to LEACH-Mobile and CBR-LEACH which has adapted TDMA to support mobile sensor in WSN [5][6]. The WSN doesn't assure the data reception to $\mathrm{CH}$ due to the mobility frequency of sensor node. In LEACH-Mobile, at the end of the frame the $\mathrm{CH}$ confirms to its member nodes that it received all data corresponding to its member nodes. All nodes must wake up before the beginning of each frame. A member node begins to transmit data in allotted time slot, if this node receives a packet delivered by $\mathrm{CH}$. If there is no reception of the packet it turns to the sleep mode until the next time slot. If there is no reception of the packet in the next time slot, it transmits a demand to join cluster because its $\mathrm{CH}$ thinks that this member node is out of the cluster. In case it doesn't acquire the transmitted data, it marks this node in the confirmation list. In the next frame, if it doesn't receive the data again from the same member node, the $\mathrm{CH}$ removes this node and it may also assign this time slot to the newly joined node in TDMA schedule.

CBR-LEACH is an advanced version of LEACH-Mobile. In CBR-LEACH the CH allocate the same time slots for the newly node which will join cluster.

In these protocols, the management of packet loss demanding a lot of additional traffic, so they consume a lot of energy compared to LEACH.

\section{B. W-LEACH}

Weighted LEACH: it's an extended LEACH [7] to challenge uniform and non-uniform network. In set up phase, the base station based on density of nodes to divide member nodes in two groups. One group member nodes transmit their data while the member nodes in the other group remain asleep. The selection of nodes which remain asleep is centralized.

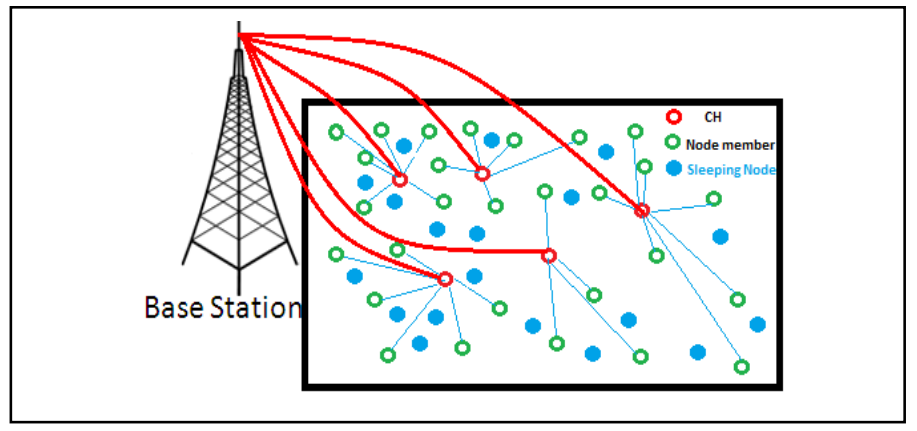

Figure 3: W-LEACH and W-LEACH Decentralized aggregation algorithms

\section{W-LEACH DECENTRALIZED}

Weighted Low Energy Adaptive Hierarchy (W-LEACH) is an extension of LEACH to efficiently handle non-uniform sensor distribution in WSNs. It increases the lifetime of the network. But this protocol is centralized because the base station is the only responsible for selecting sleeping nodes and active nodes. In addition, the base station needs the location node to manage the density of nodes after it informs each node with an additional traffic. In this paper, we propose later a decentralized algorithm which weighs LEACH without nodes' maps.

\section{A. W-LEACH decentralized details}

W-LEACH Decentralized is a decentralized algorithm. As in LEACH, W-LEACH Decentralized algorithm is divided into rounds, where each round begins with a set-up phase in which Cluster Heads $(\mathrm{CH})$ are selected and clusters are formed. Then comes the steady-state phase in which the data transmitted to the base station. Just before the beginning of data transmission, each node determines its neighbours according to a welldetermined distance which organizes the density of nodes so the neighbouring nodes do not transmit the same data. According to the number of neighbours, the node members decide their status as being in an active state or they remain in a sleep state during this round, so a sensor with low densities (has a lot of neighbours) stays alive as long as possible. In this way, the allocations of sensors densely send data to their CHs have probably leaded to minimize redundant data $\mathrm{CHs}$ and they always share in sending data with their $\mathrm{CHs}$. Thereafter the total energy consumption of the network is minimized, so we increase lifetime of network. During each round in the 
network, there are member nodes which are selected into two groups: Sleeping nodes and active nodes as is presented in Figure 3:

\section{B. W-LEACH Decentralized Algorithm}

In W-LEACH Decentralized, The selection of CHs and form clusters are as in LEACH. Each sensor node N(i) elects itself to be cluster head with a probability as presented in equation (1). In the following chart, we assume that node $\mathrm{N} 2$ is elected $\mathrm{CH}$ and $\mathrm{N} 1, \mathrm{~N} 2$ are not cluster head. So N2 spreading ADV CH to inform its neighbours that it becomes a $\mathrm{CH}$. Then N1, N3 aggregates the ADV $\mathrm{CH}$ messages from $\mathrm{CHs}$ and choose its nearest $\mathrm{CH}$. Then it transmits a JOIN REQ message to its $\mathrm{CH}$ and received JOIN REQ message from other member nodes to determine their neighbours' nodes and the distance corresponding to every neighbour. The $\mathrm{CH}$ ,in its turn, receives the JOIN REQ message to form its cluster and create TADM Schedule. Then it spreads ADVSCH message to inform there member nodes when can transmits there data. Member nodes after receiving $\mathrm{ADVSCH}$, they calculate the number of nearest neighbours to a lower maximum distance determined. If this number is less than a limited number the node goes to sleep during this round as N3. Else the nodes calculate their Time Slot to transmit their data as N1.

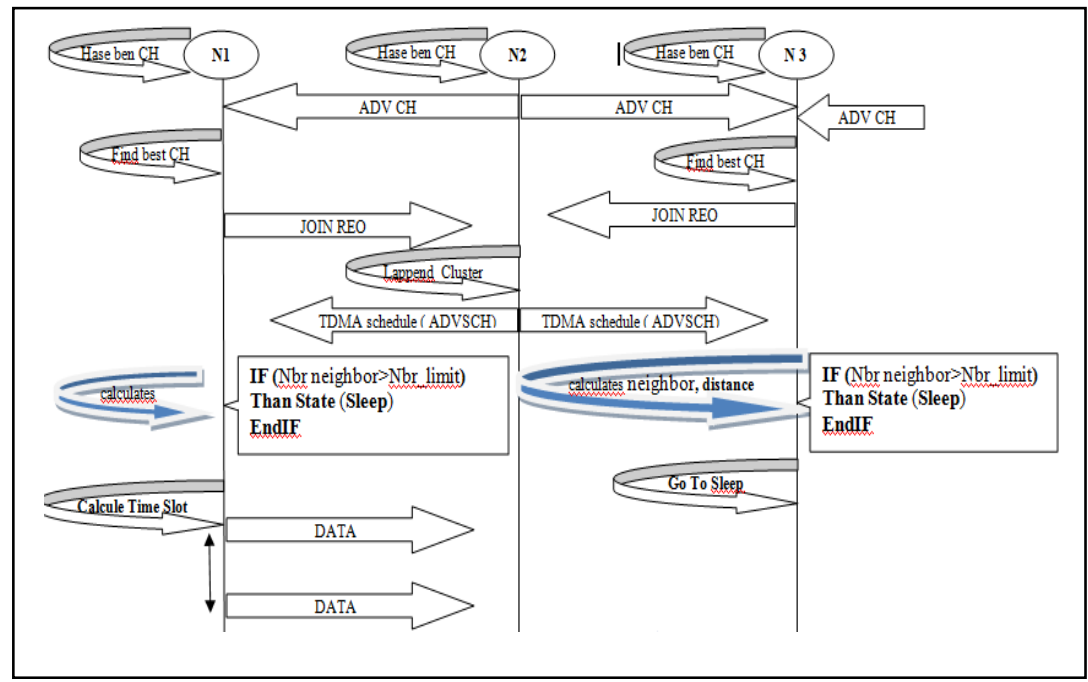

Figure 4: W-LEACH Decentralized diagram

\section{SIMULATION AND EXPERIMENTAL SETTING}

To evaluate the performance of W-LEACH Decentralized, we simulated W-LEACH Decentralized and LEACH using a network size $100 * 100 \mathrm{~m}$. A base station located at point $(70,200)$. The initial energy of each sensor is set to $2 \mathrm{~J}$. We set data size to 500 bytes. We test the algorithm with various number alive nodes: $\mathrm{n}=50$, $\mathrm{n}=100, \mathrm{n}=150, \mathrm{n}=200$. Comparative results were the average of 10 runs matching each test. We set distance_limit=5m and Number_neighbour_limit=12nodes. Simulation is carried out in NS2.34 [8] simulator with integration of LEACH protocol [9]

A. Results and discussions

We chose to track the number of nodes alive over time. In fact, it gives us an insight into the frequency of death nodes. It can be used to determine the lifetime of the network according to the above definitions: Time to death of the first node Network, Time to death of the last node Network, Time to death of a percentage of nodes in the network.

1) First node dies, Last node dies and average sensor lifetime.

The following table lists the simulation results obtained using W-LEACH Decentralized with LEACH for different total number nodes. As it's shown from the figure 5, W-LEACH Decentralized retards the times of the first, last node and average total number nodes dies, and increases the lifetime of the network when compared to LEACH.

\section{2) Number of alive sensors}

The following figures: figure $6(\mathrm{a}, \mathrm{b}, \mathrm{c}, \mathrm{d})$ illustrate the number of nodes alive over time respectively to 50 , 100, 150, 200 nodes. W-LEACH Decentralized performs better than LEACH by saving the lives of more sensors through times. So we extend the lifetime of the network. 


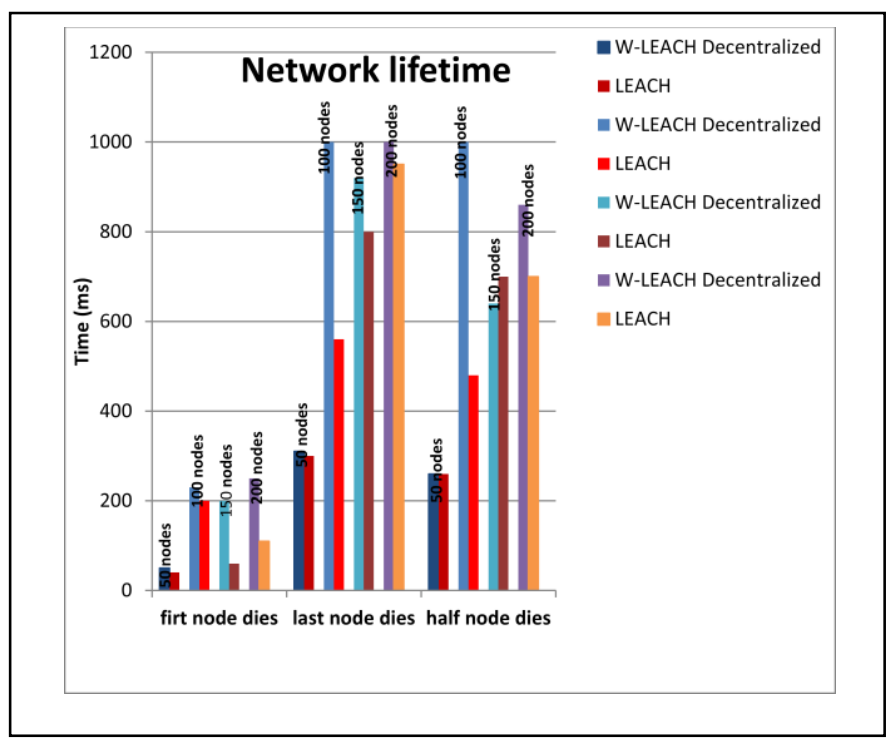

Figure 5: First node dies, last node dies and half total nodes die nodes

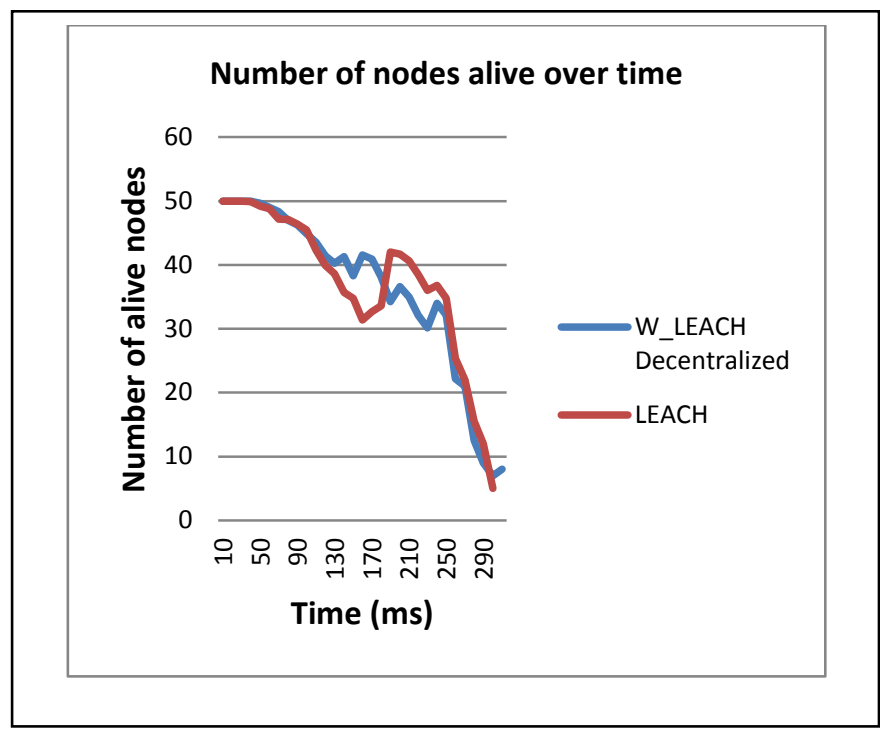

Figure 6(a): Number of nodes alive over time for 50 nodes

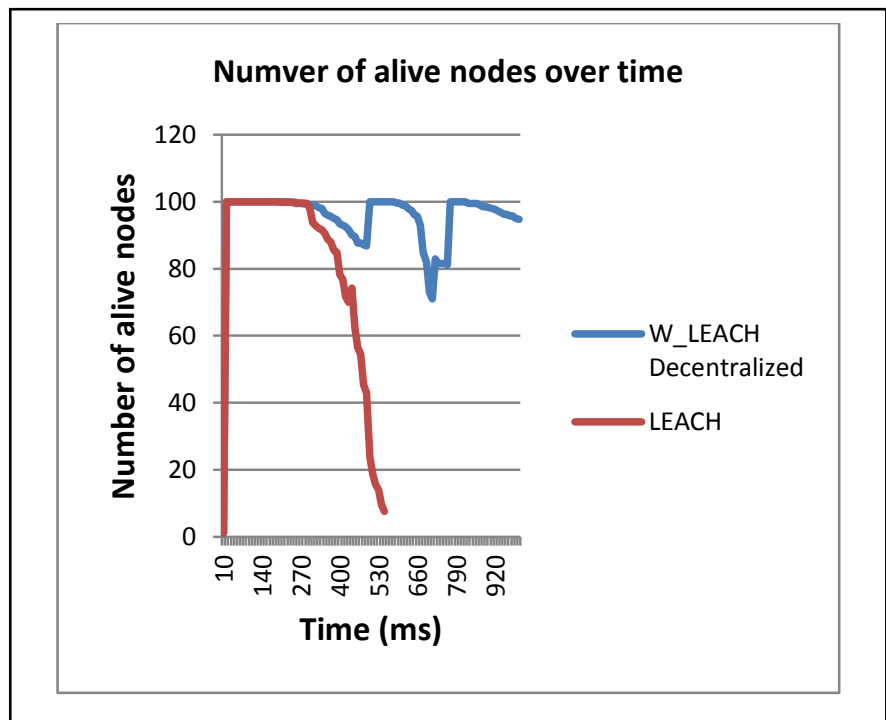

Figure 6 (b): Number of nodes alive over time for 100nodes 


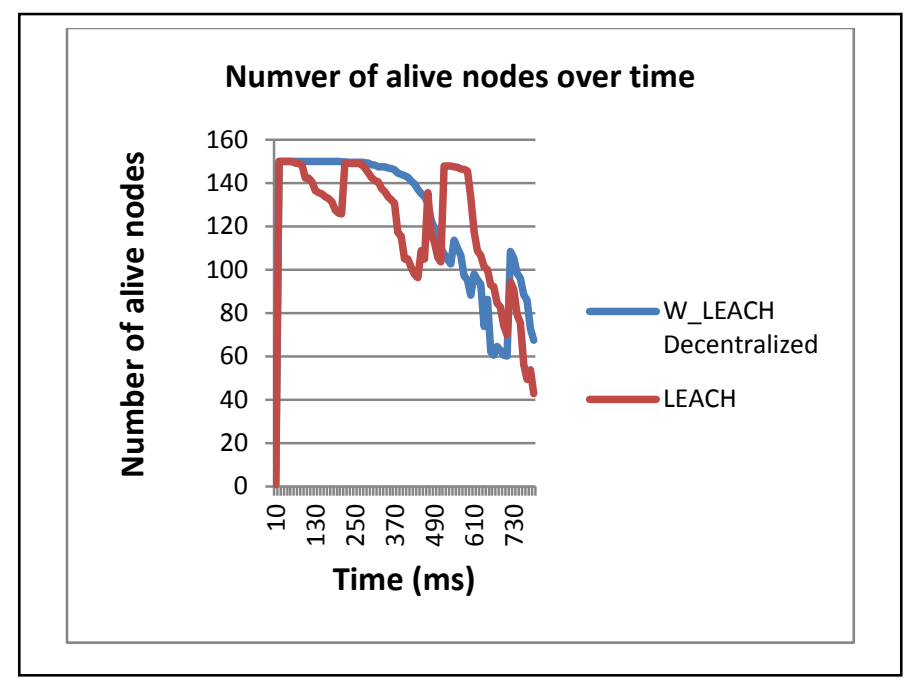

Figure 6 (c) : Number of nodes alive over time for 150nodes

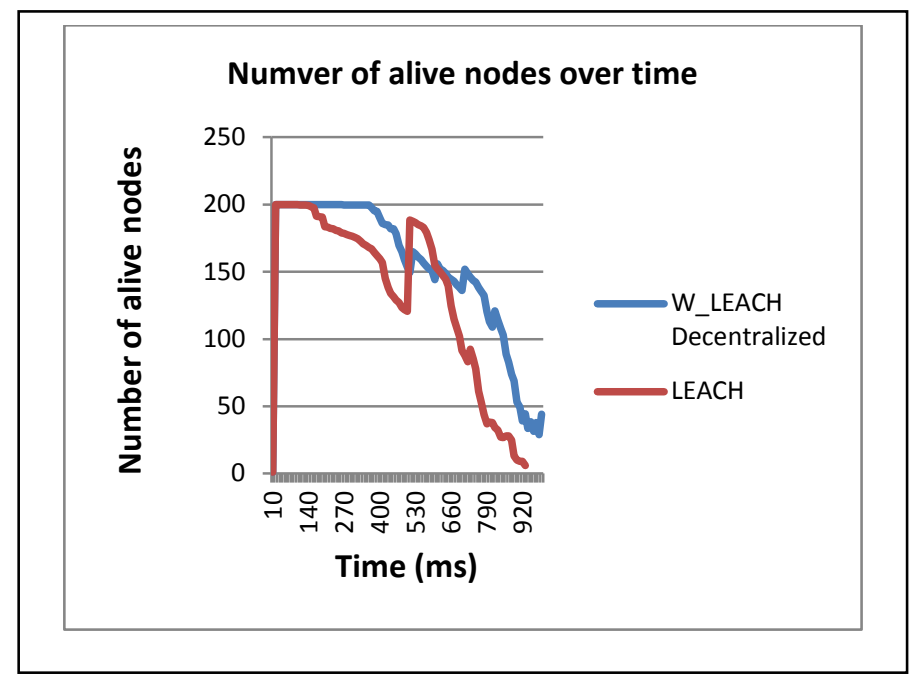

Figure 6 (d) : Number of nodes alive over time for 200

\section{3) Remaining Energy}

Figures: figure $7(\mathrm{a}, \mathrm{b}, \mathrm{c}, \mathrm{d})$ shows the total remaining energy of network versus times for W-LEACH Decentralized and LEACH respectively. It's presented clearly that the energy is saved as much as possible when W-LEACH Decentralized; therefore it operates with higher remaining energy than LEACH.

According to the preceding figures the performance of our protocol with LEACH protocol per various numbers of nodes distributed use the same area of $100 \times 100 \mathrm{~m}$ as 50,100,150 and 200. Our protocol clearly has excellent performance as compared with LEACH. When the number of nodes is equal to 50 nodes, the performance is minimal. But when the number of nodes increases, the performance is increased till it becomes maximal as presented clearly equal to 200 nodes. 
the average energy consumed over Time

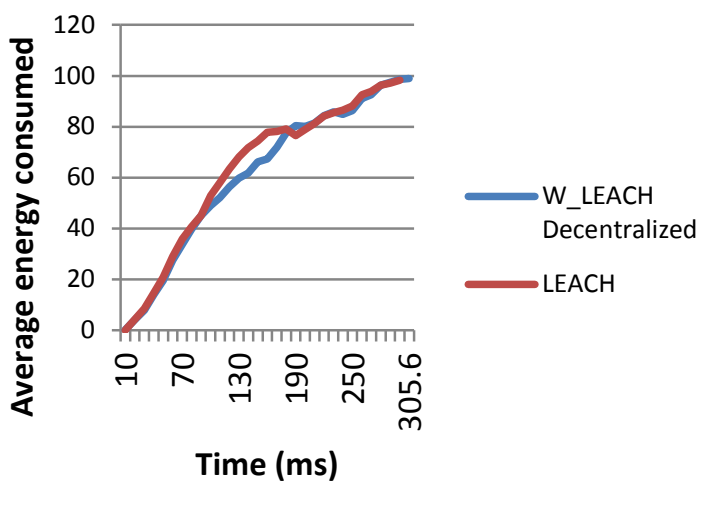

Figure 7 (a) : Energy consumed over time for 50 nodes

the average energy consumed over Time

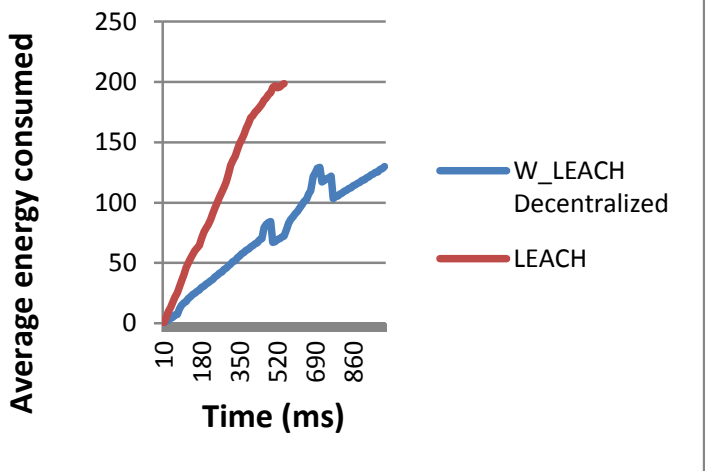

Figure 7 (b) :Energy consumed over time for 100 nodes

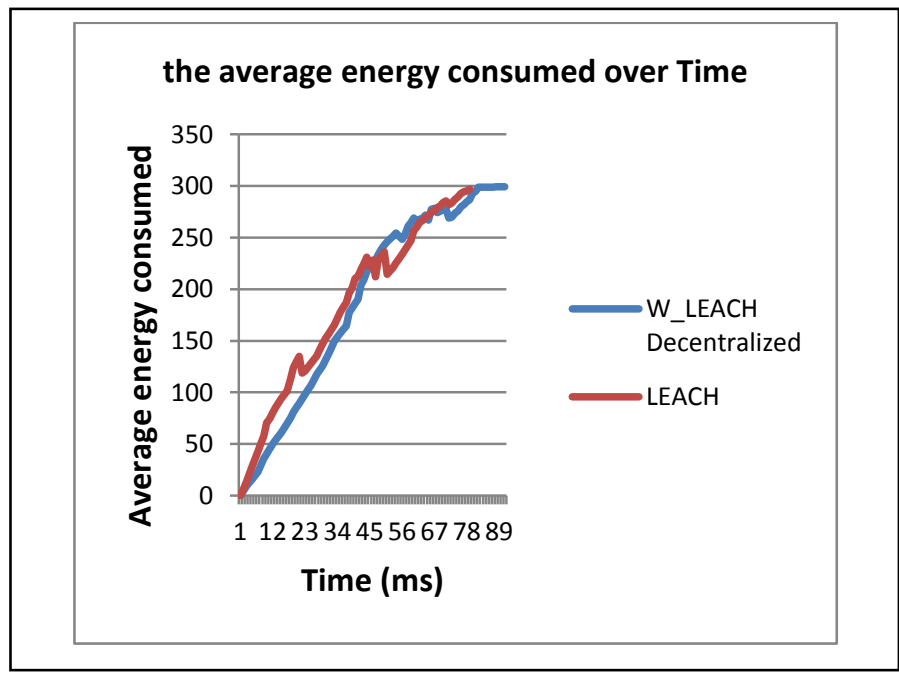

Figure 7 (c) :Energy consumed over time for 150 nodes 


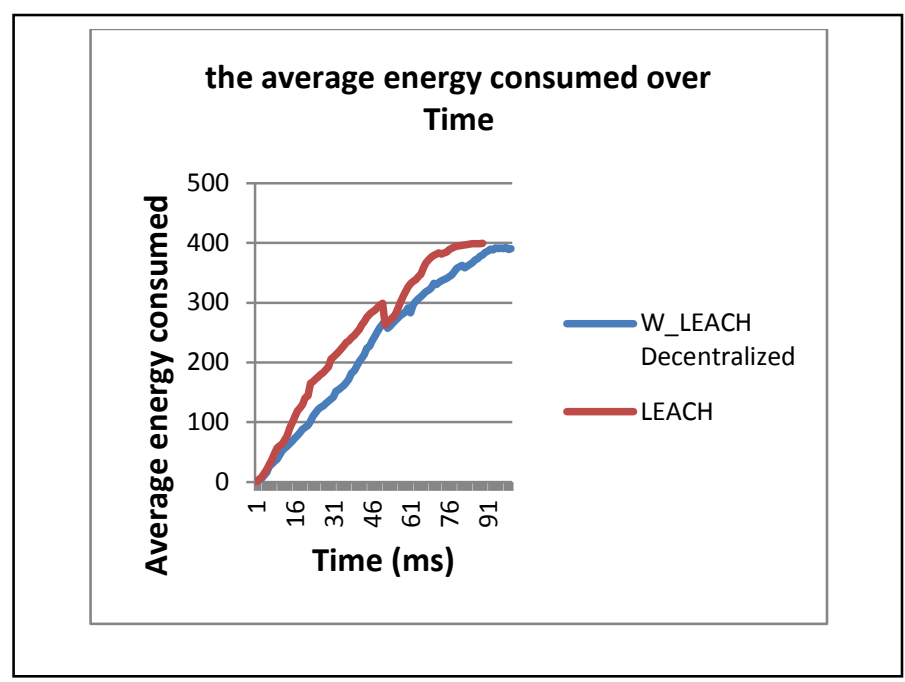

Figure 7 (d): Energy consumed over time for 200 nodes

\section{CONCLUSION AND PERSPECTIVE}

In this paper, we have described W-LEACH Decentralized; a decentralized technique to increase network lifetime of the sensor nodes in wireless sensor networks installed in the areas of large cavities abandoned mines or mapping caving. This algorithm is based on the sensor density (how many sensors are close) to put some nodes to sleep if they find a large number of neighbours. The strong point of our proposal is that it can be used in an environment where we can not determine the location of nodes. In addition, each node is able to take decision to be active or dormant autonomously. In this way, the network lifetime will be much extended. In our future work, we will test W-LEACH Decentralized with a mobile environment to consider mines or grottos environment parameters and the influences of mobility in order to control packets loss.

\section{REFERENCES}

[1] W. Heinzelman, A. Chandrakasan, H. Balakrishnan, "Energy-efficient communication protocol for wireless microsensor networks", the 33rd Hawaii International Conference on System Sciences (HICSS '00), Maui, HI, USA, 2000.

[2] W. B. Heinzelman, A. Chandrakasan, H. Balakrishnan, "An application specific protocol architecture for wireless microsensor networks," IEEE Transactions on Wireless Communications, vol. 1, pp. 660-670, Oct. 2002.

[3] G Haosong, L Gang, Y Yonghwan "A partition based centralized LEACH algorithm for wireless sensor netoworks using solar energy”, ICHIT, Korea, Aug, 2009.

[4] Nutan Sindhwani, Rohit Vaid, "V Leach: An Energy Efficient Communication Protocol For Wsn", Vol. 2, No. 2, February-March 2013, ISSN: 2320-2491

[5] D. S. Kim and Y. Chung, "Self-organization routing protocol supporting mobile nodes for wireless sensor network," in Proc. of 1st Int'1 Multi-Symposium on Computer and Computational Sciences (IMSCCS'06), 2006.

[6] S. A. B. Awwad, C. K. Ng, N. K. Noordin, and M. F. A. Rasid, "Cluster based routing protocol for mobile nodes in wireless sen sor network," in Proc. of Int'1 Symposium on Collaborative Technologies and Systems 2009, CTS'09, pp. 18-22, May 2009.

[7] Hanady M. Abdulsalam, Layla K. Kamel: W-LEACH: Weighted Low Energy Adaptive Clustering Hierarchy Aggregation Algorithm for Data Streams in Wireless Sensor Networks. ICDM Workshops 2010: 1-8

[8] Kevin Fall, Kannan Varadhan "The ns Manual” MIT, November 4, 2011.

[9] W. Heinzelman "The MIT uAMPS ns Code Extensions, Version 1.0," MIT, August 2000 